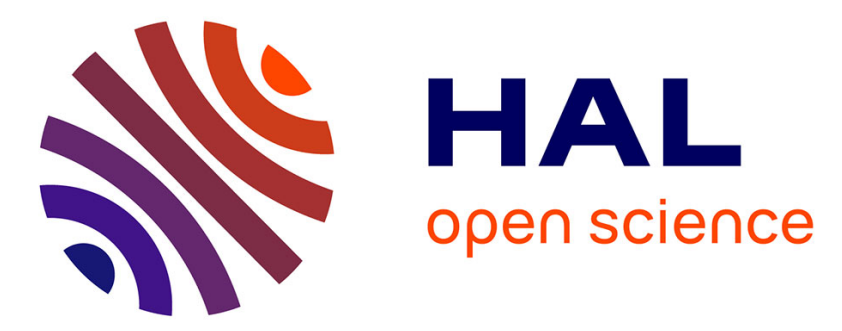

\title{
Mise en évidence des mécanismes d'injection de porteurs majoritaires à l'interface semiconducteur/électrolyte
}

J. Jaume, Catherine Debiemme-Chouvy, J. Vigneron, M. Herlem, E. Khoumri, J. Sculfort, D. Le Roy, A. Etcheberry

\section{- To cite this version:}

J. Jaume, Catherine Debiemme-Chouvy, J. Vigneron, M. Herlem, E. Khoumri, et al.. Mise en évidence des mécanismes d'injection de porteurs majoritaires à l'interface semiconducteur/électrolyte. Journal de Physique III, 1994, 4 (2), pp.273-291. 10.1051/jp3:1994129 jpa-00249102

\section{HAL Id: jpa-00249102 https://hal.science/jpa-00249102}

Submitted on 1 Jan 1994

HAL is a multi-disciplinary open access archive for the deposit and dissemination of scientific research documents, whether they are published or not. The documents may come from teaching and research institutions in France or abroad, or from public or private research centers.
L'archive ouverte pluridisciplinaire HAL, est destinée au dépôt et à la diffusion de documents scientifiques de niveau recherche, publiés ou non, émanant des établissements d'enseignement et de recherche français ou étrangers, des laboratoires publics ou privés. 


\title{
Mise en évidence des mécanismes d'injection de porteurs majoritaires à l'interface semiconducteur/électrolyte
}

\author{
J. Jaume ( $\left.{ }^{1}\right)$, C. Debiemme-Chouvy $\left({ }^{1}\right)$, J. Vigneron $\left({ }^{1}\right)$, M. Herlem $\left({ }^{1}\right)$, E. M. \\ Khoumri $\left({ }^{1}\right)$, J. L. Sculfort $\left({ }^{2}\right)$, D. Le Roy $\left({ }^{1}\right)$ et A. Etcheberry $\left({ }^{1}\right)$ \\ (1) Laboratoire de Chimie et Electrochimie de Matériaux Inorganiques, CNRS, 1 Place A. Briand, \\ 92190 Meudon, France \\ ${ }^{2}$ ) I.U.T. Troyes, Département GMP, 9 rue de Québec, 10026 Troyes Cedex, France
}

(Reçu le 14 avril 1993, révisé le 26 octobre 1993, accepté le 19 novembre 1993)

\begin{abstract}
Résumé. - La jonction semiconducteur/électrolyte est un système permettant de détecter aisément les processus d'injection de charges associées à des espèces chimiques en liaison avec le réseau cristallin. Ces phénomènes interviennent lors de réactions électrochimiques très diverses, comme les réactions de réduction multiélectroniques (oxygène, eau oxygénée) ou d'oxydation (décomposition des matériaux). Dans cet article, une comparaison entre InP et GaAs permet de discuter, dans le processus global, du rôle des intermédiaires réactionnels et des films de surface.
\end{abstract}

\begin{abstract}
The semiconductor/electrolyte junction can be used in order to detect easily electron and/or hole injection processes related to chemical species bound to the crystal lattice. These phenomena take place when multielectronic electrochemical reactions occur at the interface, such as reductions (oxygen, hydrogen peroxide) or oxidations (photoanodic decomposition). In this paper a comparison between GaAs and InP has been analyzed. The parts played in the overall electrochemical process by reaction intermediates and surface films are discussed.
\end{abstract}

\section{Introduction.}

Les études réalisées sous éclairement sur des jonctions semiconducteur(SC)/électrolyte ont mis en évidence des effets d'injection de porteurs majoritaires [1-5]. Ces processus non photoassistés caractéristiques des jonctions SC/liquide viennent s'ajouter au photocourant proprement dit. Ils induisent des effets d'amplification des courants dus aux photoporteurs minoritaires. Ces effets sont dus à des injections d'électrons $\left(\mathrm{e}^{-}\right)$en bande de conduction (BC) ou de trous $\left(\mathrm{h}^{+}\right)$en bande de valence $(\mathrm{BV})$ attribuées à la présence d'espèces intermédiaires impliquées dans le processus électrochimique global. Il est évident que ces phénomènes surviennent uniquement lors de processus électrochimiques multi-électroniques. Initialement observés sur des SC de type p, lors de photoréductions, ces effets sont à présent bien établis durant des réactions de photo-oxydation sur $\mathrm{n}-\mathrm{Si}, \mathrm{n}-\mathrm{InP}$ [6-9]. Le recours à des espèces rédox 
injectantes a permis d'établir que des effets d'injection de $\mathrm{e}^{-}$ont lieu sur $\mathrm{n}-\mathrm{GaAs}$ (très faibles), $\mathrm{n}$-GaP (faibles), et $\mathbf{n}-\mathrm{Ge}$ (faibles) lors de la réaction d'oxydation du SC à l'obscurité [10-12].

On peut classer les effets d'injection en deux catégories : les effets réversibles et les effets permanents. Les premiers peuvent être créés ou annihilés à volonté par la présence ou non d'une espèce rédox photoréactive en solution. Une telle démarche a notamment permis de mesurer les effets doubleurs présents durant la photoréduction de l'oxygène $\left(\mathrm{O}_{2}\right)$ en solution sur p-GaAs, p-GaP $[13,14]$, ainsi que sur p-CdTe [15]. Les seconds sont inhérents à la seule réaction possible au sein de l'électrolyte ; c'est le cas, par exemple, sur les systèmes non stabilisés subissant une photocorrosion. Cependant, nous verrons que l'on peut, par des changements adaptés au sein de l'électrolyte, modifier les phénomènes d'injection liés à la dissolution anodique. A ce titre des expériences relatives à la mesure d'une injection d'électrons sur une électrode partiellement stabilisée seraient très intéressantes et pourraient servir de sonde in situ pour la mesure du degré de stabilisation [16].

Dans cet article on présente différents types d'effets d'injection, en prenant appui sur l'électrochimie de matériaux III-V. et on propose une méthodologie permettant de mieux préciser l'origine de ces phénomènes.

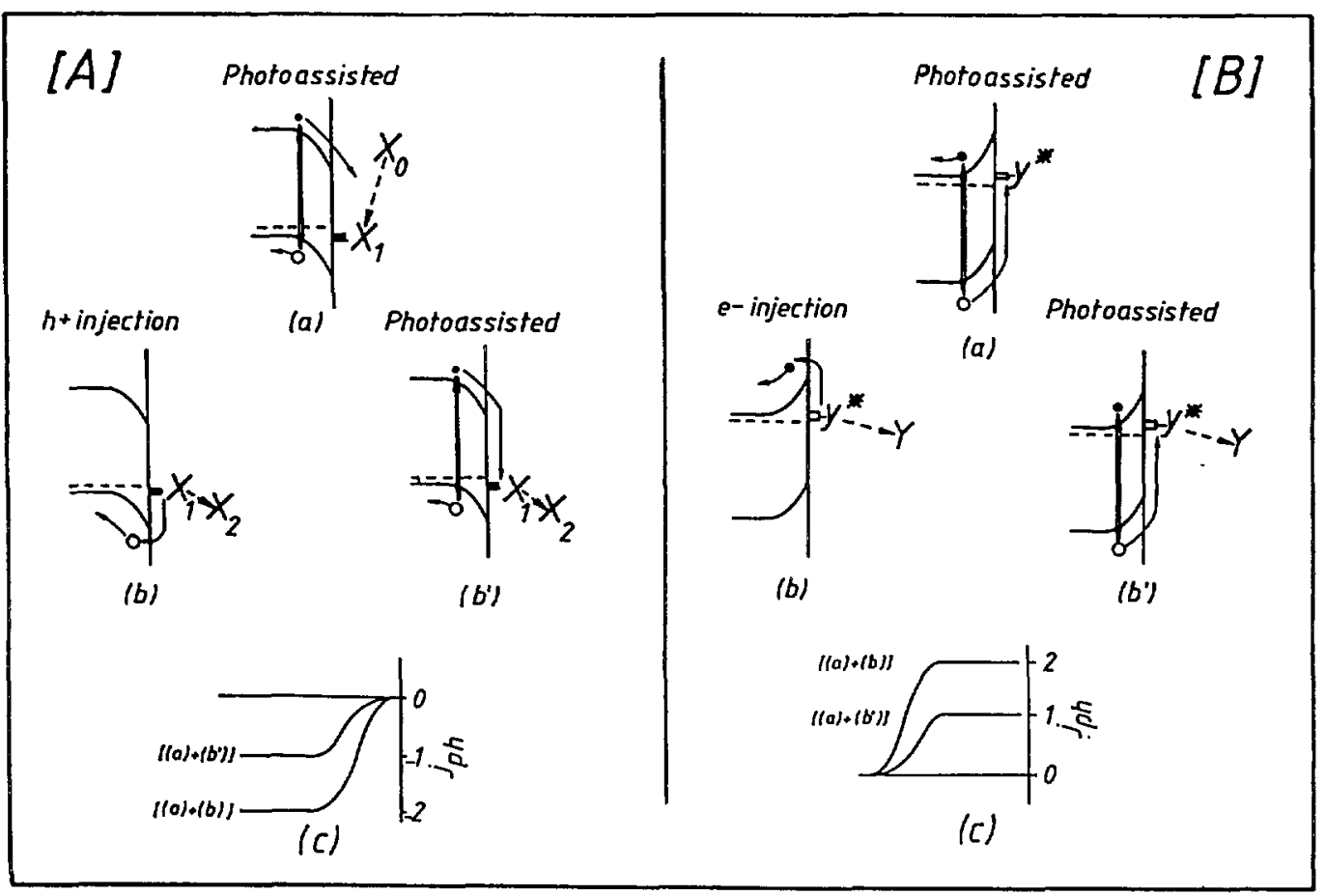

Fig. 1. - Mécanisme d'injection de porteurs majoritaires lors d'une réaction biélectronique. Le mécanisme est en deux étapes, la première est photo-assistée (a), la seconde est soit photo-assistée $\left(b^{\prime}\right)$, soit due à une injection d'un porteur majoritaire dans une des bandes (b). Courants totaux associés (c). A) Matériau de type p. B) Matériau de type n.

[Injection mechanism of majority carriers in a two-step reaction. The first step is a photoassisted reaction (a), the second is either a photoassisted reaction $\left(b^{\prime}\right)$ or an injection of the majority carriers in conduction or valence band (b). Related photocurrents (c). A) p-type sample. B) n-type sample.] 


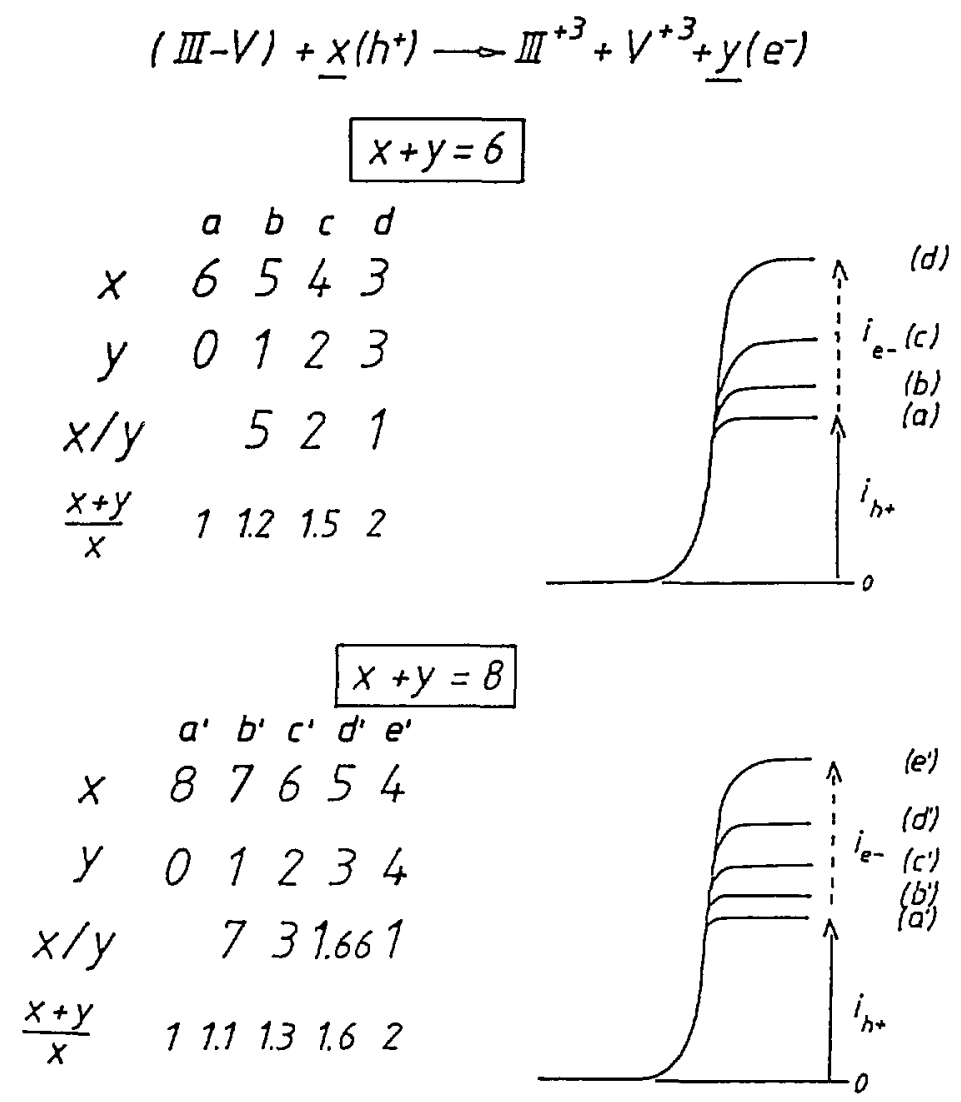

Fig. 2. - Mécanismes de dissolution anodique d'un matériau de type $\mathrm{n}$ soumis à un éclairement d'énergie supérieure à la largeur de la bande interdite $\left(h \nu>E_{\mathrm{G}}\right)$. Mécanismes impliquant 6 charges ou 8 charges. $x, y$ : nombre de trous collectés en BV et nombre d'électrons injectés en BC pour la dissolution d'une entité élémentaire III-V.

[Anodic dissolution mechanisms of a n-type illuminated sample. Light energy $h \nu>E_{\mathrm{G}}$. Overall mechanisms with 6 or 8 charges. $x, y$ : respectively number of holes collected in the VB and number of electrons injected in the $\mathrm{CB}$ for the dissolution of an elementary entity of III-V compound.]

\section{Principe et méthodologie.}

\section{PRINCIPE D'UN EFFET D'INJECTION.}

(i) Dans tous les cas l'effet d'injection du porteur majoritaire ne peut intervenir qu'après la première étape de collecte des porteurs minoritaires photogénérés. Sur la figure 1 est décrit le mécanisme général qui génère le phénomène d'injection de porteurs majoritaires à l'origine des phénomènes d'amplification des photocourants. Ces processus peuvent intervenir soit lors d'une photoréduction, soit lors d'une photo-oxydation. Par souci de simplification, nous avons schématisé le cas d'un processus rédox bi-électronique.

Pour simplifier les schémas nous avons supposé une seconde étape réalisée uniquement en terme d'injection (cas du doublement du photocourant). En réalité tout processus d'injection est en compétition avec une consommation de porteurs minoritaires dans le cadre du déroulement du processus électrochimique global. Toutes les situations entre l'amplification 
due à une (ou plusieurs) étape d'injection totale et celles liées à un processus sans injection entièrement photo-assisté peuvent être observées.

(ii) Ces dernières considérations impliquent l'existence de niveaux limites accessibles au photocourant pour un mécanisme d'injection présent dans un mécanisme global. L'accès expérimental à cette valeur limite dépendra de l'efficacité du processus d'injection par rapport à celui consommant des porteurs minoritaires photogénérés.

Pour préciser ce point nous allons particulariser, sur un exemple relatif à la dissolution anodique, la notion de limite du courant global obtenu sous illumination. Les courbes et les tableaux de la figure 2 illustrent le schéma réactionnel utilisé. Nous supposons que le niveau de photocourant de référence correspond à un processus consommant uniquement des trous $\left(i_{\mathrm{h}^{+}}\right)$. Tout effet d'injection conduira à un courant réel supérieur à ce niveau de référence. Les niveaux limites de photocourant, selon la présence d'une, de deux, etc. étapes injectantes en s'arrêtant à l'effet doubleur, sont indiqués. Ces considérations sont généralisables à tout autre cas expérimental.

Dans cet article, nous nous situerons dans le cas habituel où le photocourant est gouverné par la collecte des porteurs minoritaires à la surface et donc dépend linéairement du flux de photons incidents dont l'énergie est supérieure à la largeur de la bande interdite $\left(E_{\mathrm{G}}\right)$. Le problème des flux lumineux importants ne sera pas abordé.

\section{MÉTHOdOLOGIE POUR LA MESURE DE L'EFFET D'INJECTION.}

(i) Une interprétation rigoureuse de l'effet d'injection nécessite une mesure précise de la part due à l'injection et de celle due à la collecte des photoporteurs minoritaires dans le courant total.

Cette mesure nécessite une détermination du rendement externe, c'est-à-dire la mesure du photocourant ramenée à la mesure exacte du flux de photons pénétrant dans le SC. On peut estimer par modélisation la quantité de porteurs minoritaires arrivant à la surface et par différence donner la contribution supplémentaire non photo-assistée. Le modèle de Gartner [17] semble bien adapté à cette estimation pour des courbures de bandes suffisamment grandes pour permettre la saturation du photocourant $\left(i_{\mathrm{ph}}\right)$ sur des matériaux à transition optique directe par exemple. D'autres alternatives ont cependant été proposées [18-20]. Cette approche se heurte à des difficultés purement techniques liées à la mesure du rendement externe.

(ii) Une méthodologie alternative d'évaluation de l'effet d'injection est souhaitable. Elle nécessite d'une part une mesure du niveau d'excitation incident faisant office de référence interne et, d'autre part, une procédure expérimentale destinée à comparer, dans des conditions d'éclairement identiques, les niveaux des photocourants obtenus sur différents matériaux pour diverses réactions électrochimiques.

Le système de référence est lui-même lié à un mécanisme photo-électrochimique considéré comme reproductible, stable et parfaitement connu quant aux contributions relatives des BC et des $\mathrm{BV}$ à la photoréaction. Le système idéal ne fait intervenir que des photoporteurs minoritaires avec un rendement de collecte quasiment égal à 1 [17]. La simple comparaison des niveaux obtenus avec le niveau de référence permet alors de mettre en évidence des effets d'injection.

La quantification de ces effets est par contre plus difficile. Elle n'est a priori possible que si les conditions de collecte des porteurs minoritaires sur le matériau de référence d'une part et sur le matériau testé d'autre part sont quasi identiques. Avant toute comparaison une réflexion sur les rendements quantiques est nécessaire.

Les rendements quantiques calculés à partir du modèle de Gartner pour des valeurs caractéristiques des différents paramètres sont regroupés dans le tableau I. Dans notre cas, les coefficients d'absorption de InP et GaAs sont voisins de $\alpha=10^{5} \mathrm{~cm}^{-1}$ pour les conditions 
Tableau I. - Rendements quantiques $\eta$ calculés à partir de la formule de Gartner: $\eta=1-\exp (-\alpha W) /(1+\alpha L)[17]$, avec $\alpha$ : coefficient d'adsorption, $W$ : épaisseur de la zone de charge d'espace. $L$ : longueur de diffusion des porteurs minoritaires.

[Quantum efficiencies $\eta$ calculated using Gartner's formula $\eta=1-\exp (-\alpha W) /(1+\alpha L)$ [17], with $\alpha$ : absorption coefficient, $W:$ thickness of the depletion layer, $L:$ diffusion length of minority carriers.]

\begin{tabular}{|c|ccc|ccc|}
\hline $\begin{array}{c}N / \mathrm{cm}^{-3} \\
L / \mathrm{cm}\end{array}$ & $10^{15}$ & $10^{16}$ & $10^{18}$ & $10^{15}$ & $10^{16}$ & $10^{18}$ \\
\hline $10^{-3}$ & 0,971 & 0,936 & 0,912 & 0,999 & 0,999 & 0,993 \\
$10^{-4}$ & 0,842 & 0,652 & 0,517 & 0,999 & 0,997 & 0,936 \\
$10^{-5}$ & 0,712 & 0,368 & 0,123 & 0,999 & 0,986 & 0,652 \\
\hline & \multicolumn{3}{|c|}{$\alpha=10^{4} \mathrm{~cm}^{-1}$} & & $\alpha=10^{5} \mathrm{~cm}^{-1}$ \\
\hline
\end{tabular}

d'illumination utilisées. Les rendements quantiques sont très proches de l'unité pourvu que la longueur de diffusion $L$ soit suffisante, celle-ci dépendant de la qualité du matériau. Pour des échantillons très fortement dopés $\left(>10^{18}\right)$, une attention toute particulière devra être apportée à l'exploitation des mesures.

Dans ces conditions, la comparaison directe des niveaux des photocourants est parfaitement légitime.

\section{Conditions expérimentales et résultats.}

Les conditions et les méthodes de préparation des électrodes ont été décrites précédemment [21]. L'électrolyte est une solution aqueuse d'acide $\mathrm{H}_{2} \mathrm{SO}_{4}$ de concentrations allant de $0,5 \mathrm{M}$ à $2 \mathrm{M}$. Le taux de dopage des échantillons est compris entre $10^{15}$ et $10^{18} \mathrm{~cm}^{-3}$ Tous les potentiels sont mesurés par rapport à une électrode de référence à sulfate mercureux notée ESM.

Le montage de photocourant utilisé [21] permet une reproductibilité meilleure que $5 \%$ et une dynamique de photocourant allant de 0,1 à $100 \mu \mathrm{A} . \mathrm{cm}^{-2}$.

Pour chacune des figures, les conditions de réglage de puissance sont indiquées, ramenées à $P_{0}$, puissance lumineuse nécessaire pour obtenir une densité de courant de $10 \mu \mathrm{A} \cdot \mathrm{cm}^{-2}$ sur une électrode de InP type n.

Les figures 3-5 montrent des exemples de situations expérimentales très différentes selon la nature du matériau SC d'une même famille III-V (GaAs, InP) et selon les espèces mises en jeu dans le processus électrochimique.

Dans la figure $3 a$ sont comparés les résultats expérimentaux obtenus pour un même flux lumineux sur des électrodes de GaAs et InP monocristallines de même orientation $\langle 100\rangle$, de nombre de porteurs comparables, mais de types de conduction différents. Dans les conditions expérimentales utilisées, les quatre photocourants restent proportionnels à la puissance lumineuse du flux incident (Fig. 3b). Des photocourants anodiques sont observés pour les matériaux de type $\mathrm{n}$. Des photocourants cathodiques sont observés pour les matériaux de type $p$. Ils présentent tous un palier de saturation. Le niveau sur n-InP est nettement plus intense que ceux, assez proches, associés aux trois autres électrodes. 

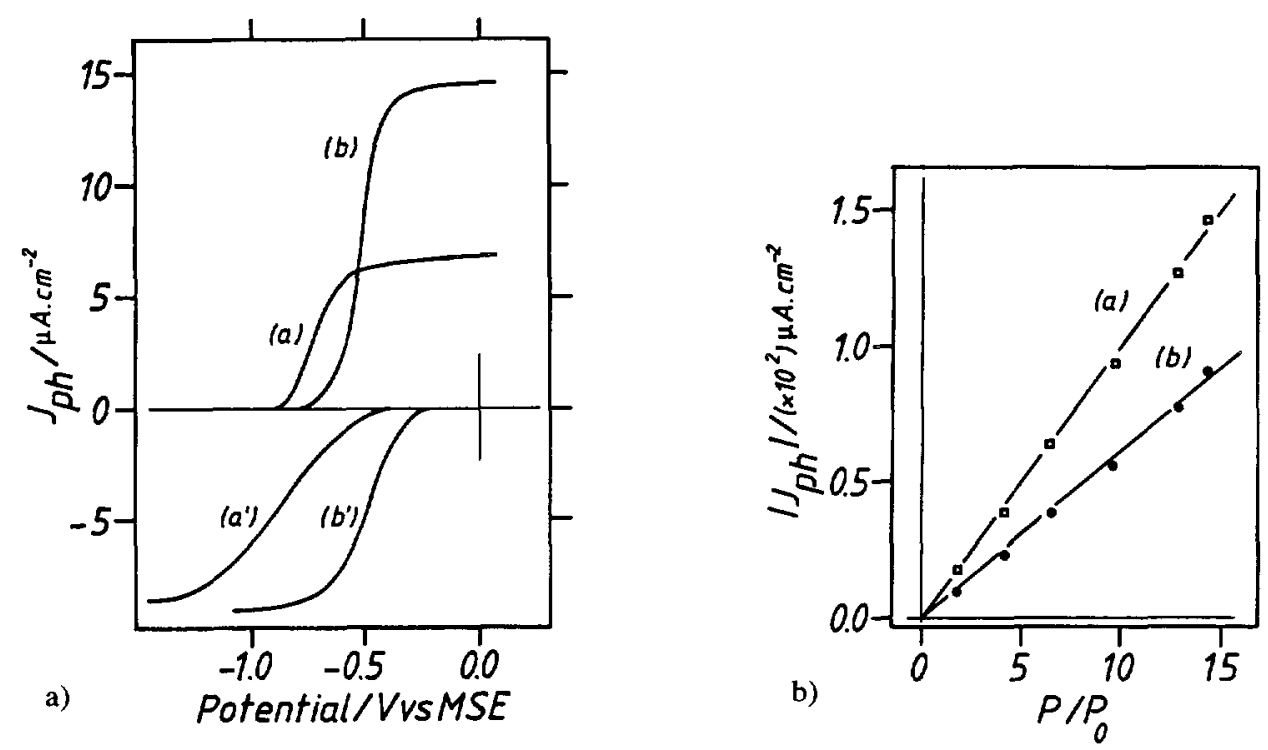

Fig. 3. - a) Photocourants expérimentaux obtenus sur différents matériaux dans les mêmes conditions d'excitation. Electrolyte : $\mathrm{H}_{2} \mathrm{SO}_{4} 0,5 \mathrm{~mol} . \mathrm{dm}^{-3} P=1,45 P_{0}$. a) GaAs de type $\mathrm{n}$. b) InP de type $\mathrm{n}$. $\left.a^{\prime}\right)$ GaAs de type $p . b^{\prime}$ ) InP de type $p$ en présence d'oxygène. b) Variations des photocourants d'électrodes de InP avec le flux lumineux : (a) InP de type $n$; (b) InP de type p. $P_{0}$. puissance lumineuse donnant une densité de courant de $10 \mu \mathrm{A} . \mathrm{cm}^{-2}$ sur une électrode de InP type $n$.

[Experimental photocurrents obtained with various compounds in the same experimental excitating conditions. Electrolyte: $\mathrm{H}_{2} \mathrm{SO}_{4} 0.5 \mathrm{~mol} . \mathrm{dm}^{-3} \quad P=1.45 P_{0}$. a) n-GaAs. b) n-InP. a') p-GaAs. $\left.b^{\prime}\right)$-InP with $\mathrm{O}_{2}$. b) Variation of photocurrents as a function of the light intensity : (a) n-InP; (b) p-InP. $P_{0}$ is the light intensity which gives a current density of $10 \mu \mathrm{A} . \mathrm{cm}^{-2}$ on $\mathrm{n}$-InP electrode.]

Les deux courants anodiques sont à relier à la photodissolution de n-GaAs et n-InP. La réaction sur $\mathrm{p}$-GaAs correspond à la photoréduction des $\mathrm{H}^{-}$Sur p-InP est montré le photocourant dû à la réduction de $\mathrm{O}_{2}$. Contrairement à p-GaAs, la photoréduction des $\mathrm{H}^{+}$n'a pas été choisie comme réaction de référence sur $\mathrm{p}$-InP du fait des difficultés à obtenir un palier de saturation bien marqué. Lorsqu'elle existe la valeur de la saturation est quasi identique à celle observée en présence de $\mathrm{O}_{2}$ [22].

Les figures 4 et 5 montrent les courants obtenus lors de la réduction d'espèces réactionnelles multiélectroniques telles que l'oxygène et l'eau oxygénée.

La figure 4 montre que l'on peut obtenir aisément en présence d'oxygène dissous une forte augmentation du photocourant sur des électrodes de p-GaAs. Cet effet est réversible, il disparaît lors du retour à une atmosphère contrôlée et inerte (argon). Ce phénomène n'est pas observé sur des électrodes de p-InP [22].

En présence d'eau oxygénée (Fig. 5a) le photocourant sur p-InP semble indépendant de la concentration en oxydant et il reste quasiment égal à celui que l'on obtient en solution désaérée d'acide ou en présence d'oxygène. Seul le potentiel d'apparition du photocourant varie avec la concentration. Par contre pour une électrode de p-GaAs (Fig. 5b), on peut observer une augmentation importante du photocourant lorsque la concentration en eau oxygénée est suffisante $\left(C>5 \times 10^{-2} \mathrm{M}\right)$. 


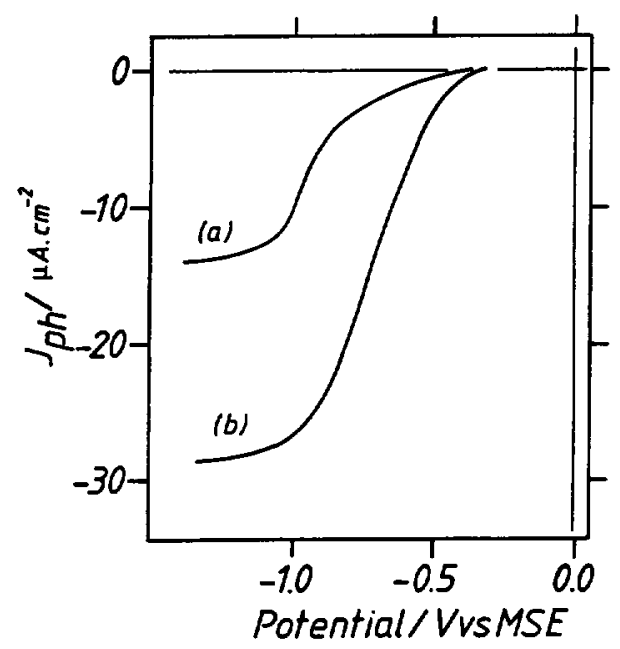

Fig. 4. - Photocourants observés sur des électrodes de $\mathrm{GaAs}$ de type $\mathrm{p}$, dans $\mathrm{H}_{2} \mathrm{SO}_{4} 0,5 \mathrm{~mol} \mathrm{dm}^{-3}$ $P=2,35 P_{0}$. a) Sans oxygène. b) En solution saturée d'oxygène.

[Photocurrents observed with p-type GaAs, $\mathrm{H}_{2} \mathrm{SO}_{4} 0.5 \mathrm{~mol} . \mathrm{dm}^{-3} P=2.35 P_{0}$. a) Without oxygen. b) In saturated oxygen solution.]

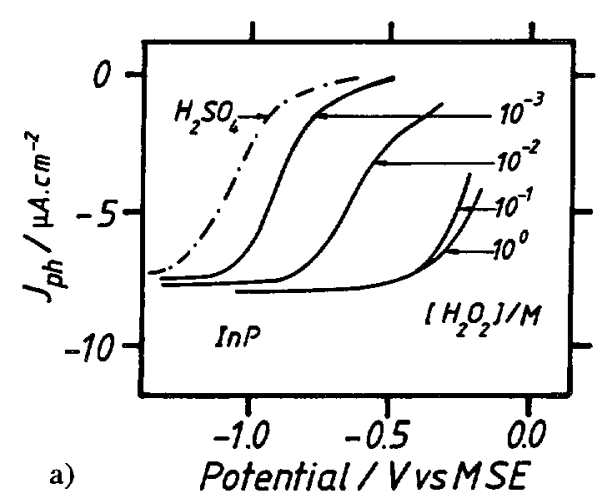

a)

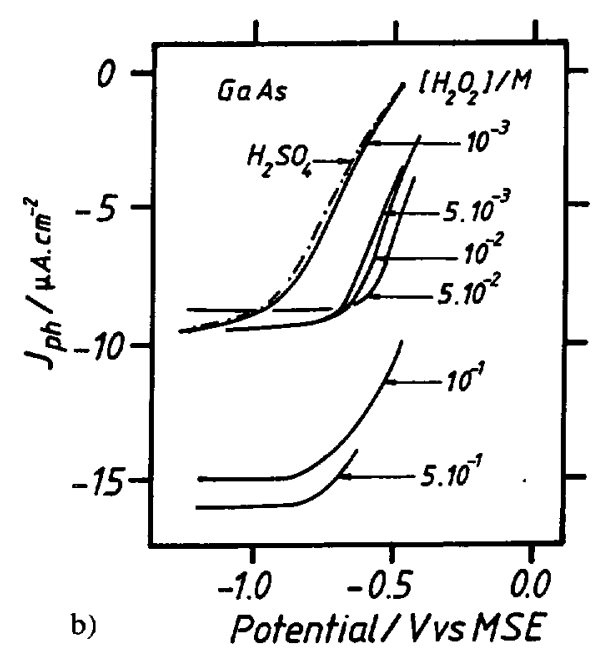

Fig. 5. - a) Photocourants obtenus sur InP de type p en présence d'eau oxygénée à des concentrations variables, dans $\mathrm{H}_{2} \mathrm{SO}_{4} 2 \mathrm{~mol} . \mathrm{dm}^{-3}(---)$ Réduction des protons. $P=1,17 P_{0}$. b) Photocourants obtenus sur GaAs de type p en présence d'eau oxygénée de concentration variable, dans $\mathrm{H}_{2} \mathrm{SO}_{4}$ $2 \mathrm{~mol} . \mathrm{dm}^{-3}(--)$ ) Réduction des protons. $P=1.6 P_{0}$.

[a) Photocurrent of p-type InP in presence of hydrogen peroxide at various concentrations, $\mathrm{H}_{2} \mathrm{SO}_{4} 2 \mathrm{~mol} . \mathrm{dm}^{-3}(--)$ ) Protons reduction. $P=1.17 P_{0}$. b) Photocurrent of p-type GaAs in presence of hydrogen peroxide at various concentrations, $\mathrm{H}_{2} \mathrm{SO}_{4} 2 \mathrm{~mol} . \mathrm{dm}^{-3}$ (- - -) Protons reduction. $\left.P=1.6 P_{0 .}\right]$ 
Ces résultats expérimentaux montrent que les surfaces des matériaux d'une même famille peuvent donner lieu à des photoréactions en apparence très diverses pour des conditions de flux lumineux très proches.

\section{Discussion.}

MISE EN ÉVIDENCE DES EFFETS D'INJECTION : CONTRIBUTION DES BANDES. - La comparaison des niveaux de saturation des photocourants observés sur les figures 3-5 fait apparaître deux types de photoréponses, les unes à bas niveau, les autres à haut niveau pour des conditions d'excitation identiques.

- On peut classer sous la première rubrique à la fois certaines photoréductions comme celle du dégagement d'hydrogène, et de la réduction de l'eau oxygénée (si $C<5 \times 10^{-2} \mathrm{M}$ )) sur des électrode de $\mathrm{p}-\mathrm{GaAs}$, celle du dégagement d'hydrogène, de la réduction de l'oxygène et de l'eau oxygénée sur des électrodes de p-InP. La photo-oxydation de n-GaAs appartient également à cette catégorie. Ces processus électrochimiques génèrent des intensités de courant relativement proches et particulièrement reproductibles qui permettent de définir un niveau plancher directement lié au niveau d'excitation imposé. Dans tous les cas, les intensités observées restent proportionnelles au flux incident.

- On peut classer dans la seconde rubrique les photoréductions de l'oxygène, de l'eau oxygénée $\left(C>5 \times 10^{-2} \mathrm{M}\right)$ sur $\mathrm{p}$-GaAs et la photo-oxydation de $\mathrm{n}$-InP. Les niveaux obtenus alors sont toujours très supérieurs aux niveaux plancher.

Les niveaux de photocourant accessibles dans cette catégorie d'expérience peuvent atteindre le double du niveau plancher tout en conservant une variation linéaire en fonction du flux.

Les effets d'amplification constatés sont associés à certains mécanismes survenant lors de photoprocessus particuliers.

Sur les figures 3-5 les niveaux peuvent être ramenés à un même flux lumineux par une simple correction de puissance, ce qui permet de situer les différents mécanismes d'amplification du photocourant les uns par rapport aux autres.

Les conditions de linéarité en flux étant respectées, on peut supposer, sans faire référence à une modélisation que le facteur limitant est le processus de collecte des photoporteurs. Les courbures de bandes et les propriétés physiques étant très proches (entre InP et GaAs), il est normal d'obtenir des niveaux plancher de saturation très proches pour ces deux matériaux. Ce niveau peut alors être considéré comme une caractéristique des processus associés uniquement aux photoporteurs. Il peut alors être utilisé comme le niveau de référence des photoporteurs.

L'amplification du photocourant par rapport à ce niveau plancher de référence implique que des porteurs non-photogénérés participent au processus global de réduction ou d'oxydation. Les chemins de transfert associés peuvent être dus soit à une injection d'électrons dans la bande de conduction survenant lors d'une oxydation, soit à une injection de trous dans la bande de valence survenant lors d'une réduction.

Les fortes variations des photocourants observés dans certains cas peuvent être directement imputées aux modulations des effets d'injection de porteurs non photogénérés.

Pour un photocourant donné, on peut ainsi déterminer conformément au tableau II les contributions respectives de la $\mathrm{BV}$ et de la $\mathrm{BC}$ dans chaque mécanisme réactionnel.

- La réduction des protons est une réaction à deux étapes. Les figures 3-5 montrent que les deux étapes sont entièrement photo-assistées sur les électrodes de p-GaAs et p-InP (réaction 11 Tabl. II).

- Pour la réduction de l'oxygène sur les électrodes de p-InP, le transfert électronique se fait également par la $\mathrm{BC}$ puisque l'injection des trous a été évaluée au maximum à $3 \%$ du niveau global (Tabl. II réaction 2-1) [22]. A l'opposé, sur une électrode de p-GaAs, la photoréduction 
Tableau II. - Mécanismes réactionnels possibles pour certaines réactions électrochimiques multi-électroniques.

[Possible reaction mechanisms for certain multielectronic electrochemical reactions.]

Toutes ces réactions ont lieu en milieu aqueux acide. Toutes ces réactions sont multiélectroniques.

\section{DÉGAGEMENT DE L'HYDROGÈNE}

Réaction globale :

$$
2 \mathrm{H}^{+}+2 \mathrm{e}^{-} \rightarrow \mathrm{H}_{2}
$$

Mécanisme possible 1: intervention unique de la $B C$

$$
\begin{aligned}
\mathrm{H}^{+}+\mathrm{e}^{-} & \rightarrow \mathrm{H}_{\mathrm{ads}} \\
\mathrm{H}^{+}+\mathrm{H}_{\mathrm{ads}}+\mathrm{e}^{-} & \rightarrow \mathrm{H}_{2} .
\end{aligned}
$$

Mécanisme possible 2 : intervention des deux bandes

$$
\begin{gathered}
\mathrm{H}^{+}+\mathrm{e}^{-} \rightarrow \mathrm{H}_{\mathrm{ads}} \\
\mathrm{H}_{\mathrm{ads}}+\mathrm{H}^{+} \rightarrow \mathrm{H}_{2}+\mathbf{h}^{+}
\end{gathered}
$$

\section{RÉDUCTION DE L'OXYGÈNE}

Réaction globale à 4 électrons :

$$
\mathrm{O}_{2}+4 \mathrm{e}^{-}+4 \mathrm{H}^{+} \rightarrow 2 \mathrm{H}_{2} \mathrm{O} \text {. }
$$

Réaction globale à 2 électrons :

$$
\mathrm{O}_{2}+2 \mathrm{e}^{-}+2 \mathrm{H}^{+} \rightarrow \mathrm{H}_{2} \mathrm{O}_{2} \text { si arrêt à } \mathrm{H}_{2} \mathrm{O}_{2} \text {. }
$$

Mécanismes pour les $1^{\text {res }}$ et $2^{e}$ étapes:

Mécanisme possible 1 : intervention unique de la $B C$

$$
\begin{aligned}
\mathrm{O}_{2}+\mathrm{e}^{-}+\mathrm{H}^{+} & \rightarrow \mathrm{HO}_{2} \\
\mathrm{HO}_{2}+\mathrm{H}^{+}+\mathrm{e}^{-} & \rightarrow \mathrm{H}_{2} \mathrm{O}_{2} .
\end{aligned}
$$

Mécanisme possible 2 : intervention des deux bandes

$$
\begin{aligned}
\mathrm{O}_{2}+\mathrm{e}^{-}+\mathrm{H}^{+} & \rightarrow \mathrm{HO}_{2} \\
\mathrm{HO}_{2}^{+}+\mathrm{H}^{+} & \rightarrow \mathrm{H}_{2} \mathrm{O}_{2}+\mathrm{h}^{+}
\end{aligned}
$$

Mécanismes pour les $3^{e}$ et $4^{e}$ étapes : voir la réduction de l'eau oxygénée en considérant que chacune de ces étapes peut alors faire intervenir un trou. 


\section{RÉDUCTION DE L'EAU OXYGÉNÉE}

Réaction globale:

$$
\mathrm{H}_{2} \mathrm{O}_{2}+2 \mathrm{e}^{-}+2 \mathrm{H}^{+} \rightarrow 2 \mathrm{H}_{2} \mathrm{O} \text {. }
$$

Mécanisme possible 1 : intervention unique de la $B C$

$$
\begin{aligned}
\mathrm{H}_{2} \mathrm{O}_{2}+\mathrm{H}^{+}+\mathrm{e}^{-} & \rightarrow \mathrm{OH}^{\circ}+\mathrm{H}_{2} \mathrm{O} \\
\mathrm{OH}^{\prime}+\mathrm{H}^{+}+\mathrm{e}^{-} & \rightarrow \mathrm{H}_{2} \mathrm{O} .
\end{aligned}
$$

Mécanisme possible 2 : intervention des deux bandes

$$
\begin{aligned}
\mathrm{H}_{2} \mathrm{O}_{2}+\mathrm{H}^{+}+\mathrm{e}^{-} & \rightarrow \mathrm{OH}^{+}+\mathrm{H}_{2} \mathrm{O} \\
\mathrm{OH}^{-}+\mathrm{H}^{+} & \rightarrow \mathrm{H}_{2} \mathrm{O}+\mathrm{h}^{+}
\end{aligned}
$$

\section{PHOTODÉCOMPOSITION ANODIQUE DU MATÉRIAU}

Réaction globale :

$$
\begin{gathered}
\mathrm{III}-\mathrm{V}+x \mathrm{~h}^{+} \rightarrow \mathrm{III}^{3+}+\mathrm{V}^{3+\text { ou } 5+}+y \mathrm{e}^{-} \\
x+y=6 \text { si } \mathrm{V}^{3+} ; \quad x+y=8 \quad \text { si } \quad \mathrm{V}^{5+}
\end{gathered}
$$

Mécanisme possible 1 : intervention unique de la $B V$

$$
\mathrm{III}-\mathrm{V}+(6 \text { ou } 8) h^{+} \rightarrow \mathrm{III}^{3+}+\mathrm{V}^{3+\text { ou } 5+}
$$

Mécanisme possible 2 : intervention des deux bandes : une ou plusieurs étapes d'injection d'électrons (cas b, $\mathrm{d}^{\prime}$ et $\mathrm{d}$ de la figure 2)

$$
\begin{array}{llll}
2 \mathrm{a} & x+y=6: & \mathrm{III}-\mathrm{V}+5 h^{+} \rightarrow \mathrm{III}^{3+}+\mathrm{V}^{3+}+\mathrm{e}^{-} \\
2 \mathrm{~b} & x+y=8: & \mathrm{III}-\mathrm{V}+5 h^{+} \rightarrow \mathrm{III}^{3+}+\mathrm{V}^{5+}+3 \mathrm{e}^{-} \\
2 \mathrm{c} & x+y=6: & \mathrm{III}-\mathrm{V}+3 h^{+} \rightarrow \mathrm{III}^{3+}+\mathrm{V}^{3+}+3 \mathrm{e}^{-}
\end{array}
$$

de l'oxygène déclenche un fort processus d'injection de trous en BV (Fig. 4) (réaction 2-2). Les BV et BC sont donc impliquées. L'intensité de l'effet d'injection dépend du flux lumineux [13].

- La photoréduction de l'eau oxygénée peut être considérée comme entièrement photoassistée tant sur p-InP que sur p-GaAs (réaction 3-1). Cependant, dans le cas de p-GaAs, l'augmentation de la concentration en eau oxygénée à des valeurs supérieures à $5 \times 10^{-2} \mathrm{M}$ déclenche progressivement l'apparition d'un effet d'injection (Fig. 5b) (réaction 3-2) ce qui démontre une contribution progressive de la $\mathrm{BV}$.

- Lors de la photo-oxydation des matériaux III-V, le mécanisme est presque totalement photo-assisté pour les électrodes de n-GaAs (réaction 4-1) et partiellement photo-assisté pour les électrodes de n-InP (réaction 4-2). En effet, dans ce cas une forte contribution de la bande de conduction doit être envisagée puisque une ou plusieurs étapes d'injection d'électrons sont mises en évidence. 
NiveAu DE L'inJection: DÉTERMination DES MÉCANismes RÉACTIONNELS. - Après sa détection, la quantification de l'effet d'injection permettra de déterminer partiellement voire totalement certains des chemins réactionnels de l'ensemble du processus.

Photoréduction de $\mathrm{H}_{2} \mathrm{O}_{2}$ et $\mathrm{O}_{2}$ sur p-GaAs. - Sur p-GaAs le changement de mécanisme réactionnel (eau oxygénée-proton) ou (oxygène-proton) permet de passer réversiblement d'une situation d'injection à celle d'un processus uniquement photo-assisté.

En présence de $\mathrm{H}_{2} \mathrm{O}_{2}$ un effet doubleur est observé à forte concentration. Le caractère biélectronique du mécanisme réactionnel implique une seconde étape entièrement injectante (réaction 2-2, Tabl. II). Ce caractère totalement injectant disparaît progressivement lorsque l'on abaisse la concentration. Dans ce cas lors de la seconde étape la participation au transfert de la $\mathrm{BV}$ diminue au profit de la $\mathrm{BC}$. L'analyse du niveau d'injection permet de quantifier l'importance relative des mécanismes 2-1 et 2-2 décrits dans le tableau II.

Dans le cas de la réduction de l'oxygène sur $\mathbf{p}$-GaAs l'effet doubleur implique que le nombre de trous injectés en $\mathrm{BV}$ et le nombre d'électrons photogénérés sont égaux.

$\mathrm{Si}$ la réduction de $\mathrm{O}_{2}$ s'arrête à la formation de $\mathrm{H}_{2} \mathrm{O}_{2}$, processus à 2 électrons, l'effet d'injection prend alors place dans la seconde étape. Si la réduction se poursuit jusqu'à la formation de $\mathrm{H}_{2} \mathrm{O}$, un processus à 4 électrons, l'injection peut a priori se produire dans l'une des trois dernières étapes. Cependant le caractère photoassisté de la réduction de $\mathrm{H}_{2} \mathrm{O}_{2}$ exclut toute injection lors de la troisième étape. L'injection peut donc se produire soit lors de la deuxième étape soit lors de la quatrième.

Les résultats sur la photoréduction de $\mathrm{H}_{2} \mathrm{O}_{2}$ permettent d'une part de choisir entre un mécanisme à 2 ou à 4 électrons et d'autre part de situer l'étape injectante. En effet, les solutions saturées en oxygène ayant une concentration voisine de $10^{-3} \mathrm{~mol} . \mathrm{dm}^{-3}$, il est certain que la concentration superficielle d'eau oxygénée formée est largement inférieure à la valeur critique à partir de laquelle l'effet doubleur observé sur p-GaAs lors de la photoréduction de l'eau oxygénée seule, apparaît. On peut alors localiser dans le cas d'un processus à quatre êlectrons, la seule étape d'injection possible au niveau de la réduction du radical $\mathrm{HO}_{2}^{\circ}$. Les trois autres étapes sont par conséquent entièrement photo-assistées. Une telle situation ne peut en aucun cas aboutir à un effet doubleur. En effet, une simple analyse du courant limite accessible dans un tel cas montre que l'on peut atteindre au maximum une valeur $(x+y) / x$ égale à 1,33 $(x=3, y=1)$ fois celle du niveau plancher relatif à un processus totalement photo-assisté $(y=0)$. L'écart avec le niveau doubleur observé rend incohérente l'hypothèse d'un mécanisme de réduction de l'oxygène sur $\mathrm{p}$-GaAs à quatre étapes. Le processus doit nécessairement s'arrêter à la formation de l'eau oxygénée. Ceci est en parfait accord avec les suppositions faites dans la littérature [13].

Photo-oxydation de n-InP.

(i) Quantification de l'effet d'injection. - Dans ce cas la mesure de l'effet d'injection ne peut être qu'indirecte du fait de l'impossibilité de substituer à la réaction de dissolution, une oxydation d'espèce électroactive intentionnellement introduite en solution. Le mécanisme n'est pas modifiable et seule la comparaison avec un niveau de référence associé à un autre transfert totalement photo-assisté peut permettre la mesure du niveau d'injection. Cette référence choisie on pourra évaluer les grandeurs caractéristiques de l'effet d'injection sur $n$ InP à savoir $I(\operatorname{Ref}) /(I(\mathrm{n}-\mathrm{InP})-I(\operatorname{Ref}))$ et $I(\mathrm{n}-\mathrm{InP}) / I(\mathrm{Ref})$. La première représente le rapport entre la contribution par porteurs minoritaires et celle liée à l'injection, la seconde décrit le facteur d'amplification dû à l'injection.

L'examen de la figure 3 montre que la photo-oxydation de $n$-GaAs ainsi que les photoréductions des protons et de l'oxygène respectivement sur $\mathrm{p}$-GaAs et $\mathrm{p}$-InP peuvent être 
Tableau III. - Grandeurs expérimentales caractéristiques de l'effet d'injection, obtenues à partir des courbes de la figure 3a.

[Experimental parameters due to injection effect, obtained from curves in figure 3a.]

\begin{tabular}{|c|c|c|c|}
\hline Ref & $\mathrm{p}-$ InP & $\mathrm{p}$-GaAs & $\mathrm{n}$-GaAs \\
\hline$\frac{I(\text { Ref })}{I(\mathrm{n}-\text { InP })-I(\text { Ref })}$ & 1,60 & 1,37 & 0,96 \\
\hline$\frac{I(\mathrm{n}-I n \mathrm{P})}{I(\text { Ref })}$ & 1,63 & 1,73 & 2,04 \\
\hline
\end{tabular}

prises comme référence. Les grandeurs caractéristiques obtenues à partir de ces trois niveaux sont reportées dans le tableau III.

- Référence : n-GaAs. - L'utilisation de n-GaAs comme référence conduit à des valeurs moyennes de 0,96 et 2,04 (Tabl. III) qui diffèrent notablement des autres résultats.

La valeur de saturation du photocourant sur n-GaAs est sujette, suivant l'échantillon utilisé, à de légères variations qui dépendent du taux de dopage et de la préparation de la surface de l'électrode. Par ailleurs les rapports $I(\mathrm{n}-\mathrm{InP}) / I(\mathrm{n}-\mathrm{GaAs})$ indiqués dans la littérature varient entre 1,65 et $2,10[23,24]$. Ces considérations nous incitent à penser que les niveaux d'injection sur $\mathrm{n}$-InP, déterminés en utilisant $\mathrm{n}$-GaAs comme référence, fluctuent et sont moins fiables.

- Référence : p-GaAs ou p-InP. - Les estimations de l'effet d'injection obtenues à partir de $\mathrm{p}$-GaAs et de $\mathrm{p}$-InP sont très proches. Sur p-GaAs, nous avons effectué des mesures sur des électrodes issues d'une même plaquette donc sur un seul taux de dopage. On a remarqué que les niveaux sont plus reproductibles et plus intenses que ceux obtenus sur les types $\mathrm{n}$. Ceci explique que les estimations des injections faites à partir de p-GaAs sont nettement plus proches de celles réalisées à partir de p-InP.

Les valeurs obtenues à partir de p-InP sont très reproductibles. La diversité des échantillons utilisés, tant en type-n qu'en type-p, donnent aux valeurs associées au couple (n-InP/p-InP) un caractère de fiabilité supérieur à celui des autres mesures. On remarque que c'est dans ce cas que l'estimation de l'injection est minimale bien que toujours très intense.

D'une manière générale les variations des niveaux de saturation liés aux mécanismes entièrement photo-assistés peuvent avoir plusieurs origines. Il peut s'agir de variations des propriétés optiques de surface, de différences dans les rendements de collecte, de problèmes de chute de rendement de collecte (pour $h \nu \gg E_{\mathrm{G}}$ ) caractéristiques des jonctions électrolytiques.

Le premier point difficile à évaluer ne doit pas être écarté a priori. Le deuxième est pris en compte par le modèle de Gartner. Il est probable que des effets de baisse de rendement, non prévus par ce modèle, soient à l'origine des fluctuations observées entre niveaux plancher de matériaux et/ou de types différents. Ces effets de chute de rendement pour des énergies de photons incidents croissantes ne sont que très partiellement expliquées à ce jour [24], ils peuvent être appréhendés par une approche spectrale [25].

Compte tenu de l'ensemble des remarques ci-dessus, on peut considérer que le choix comme référence de p-InP pour mesurer quantitativement le taux d'injection sur n-InP est le plus judicieux. Il élimine les effets optiques de surface, il garantit des propriétés optiques et électriques en volume quasi identiques et l'on peut raisonnablement supposer des pertes de rendement en fonction de la longueur d'onde assez proches.

(ii) Interprétation de l'effet d'injection. - Les tableaux de la figure 2 résument aussi la discussion sur la signification que l'on doit donner à l'amplitude du niveau d'injection observé 
pendant la photo-dissolution de n-InP. Dans ces tableaux sont pris en compte les deux mécanismes réactionnels les plus probables pour décrire la dissolution d'un composé III-V. La différence réside dans le nombre de charges globalement impliquées, qui conditionne le degré d'oxydation final de l'élément $\mathrm{V}$.

Pour chaque mécanisme nous avons simulé différentes configurations des phénomènes d'injection. Nous avons supposé sur une ou plusieurs étapes un transfert engendré uniquement par injection d'électrons en bande de conduction tandis que les autres étapes consommaient uniquement des phototrous.

On peut ainsi analyser les conséquences de telles modifications sur les grandeurs caractéristiques de l'effet d'injection que sont les rapports $x / y$ et $(x+y) / x$ (Fig. 2) qui correspondent aux valeurs limites que peuvent atteindre, dans chaque cas, les grandeurs expérimentales $i_{h^{+}} / i_{f^{-}}=I(\operatorname{Ref}) /(I(\mathrm{n}-\mathrm{InP})-\mathrm{I}(\operatorname{Ref}))$ et $\left(i_{h^{+}}+i_{e^{-}}\right) / i_{h^{+}}=I(\mathrm{n}-\mathrm{InP}) / I(\operatorname{Ref})$.

On remarque que selon le mécanisme global, selon le nombre d'étapes totalement injectantes, les grandeurs $x / y$ et $(x+y) / x$ prennent des valeurs caractéristiques. En supposant que l'on puisse atteindre expérimentalement un tel régime limite, on peut alors par simple détermination du niveau d'injection sélectionner à la fois le mécanisme global et le nombre d'étapes injectantes. Cette sélectivité disparaît bien évidemment en l'absence d'injection et partiellement en cas d'effet doubleur où la séparation entre 6 et 8 charges globales par équivalent-molécule est alors impossible.

L'analyse de l'ensemble des valeurs expérimentales indique que le niveau d'injection est très élevé. L'amplitude expérimentale est telle que l'on doit supposer, sans autre analyse, que les niveaux d'amplification des photocourants expérimentaux sont voisins de ceux prévus par simulation dans l'hypothèse d'un nombre d'étapes injectantes important. L'intérêt d'une simulation des cas limites pour interpréter les observations expérimentales est évident, car la probabilité d'émission d'électrons à certains stades du processus global est très forte, voire égale à 1 .

Si l'on considère que le facteur d'amplification de 1,63 obtenu sur la base de la comparaison $\mathrm{n}$-InP/p-InP est le plus réaliste, on peut préciser parmi l'ensemble des mécanismes proposés ceux qui peuvent convenir à une telle valeur. On supposera dans un premier temps l'existence d'un mécanisme où toutes les étapes injectantes ont une probabilité d'émission d'électrons égale à 1 . Dans ce cas la valeur de 1,63 privilégie un mécanisme à 8 charges globales constitué de 5 étapes par trous et de trois étapes d'injection d'électrons (Tabl. Fig. 2). Si l'on suppose qu'aucune étape ne soit à même d'être purement injectante le facteur expérimental d'amplification doit être inférieur au facteur limite correspondant. Dans ce cas les mécanismes à 6 ou 8 charges avec respectivement 3 ou 4 étapes injectantes peuvent être pris en considération. Notons que la faible dépendance du facteur d'amplification avec la puissance lumineuse sur la gamme d'excitation présentée dans la figure $4 \mathrm{~b}$ rend moins probable cette seconde configuration. Néanmoins une réponse à cette question nécessiterait une détermination précise du facteur d'amplification sur une gamme de puissance plus étendue.

ORIGINE DE L'INJECTION : RÔLE DE LA CHIMIE DE SURFACE. - Les niveaux d'injection fort différents observés pour des matériaux de structures très proches montrent que cette injection est un phénomène spécifique de l'interface $\mathrm{SC} /$ solution. La première étape du processus correspond toujours à la capture d'un porteur minoritaire. Les étapes suivantes peuvent donner lieu à deux mécanismes cinétiquement concurrentiels : capture d'un porteur minoritaire ou injection d'un porteur majoritaire conduisant à une augmentation plus ou moins importante, selon le nombre d'étapes d'injection, du photocourant expérimental.

$\mathrm{Au}$ cours de la réaction l'interface est modifiée faisant apparaître à chaque étape des intermédiaires réactionnels et de nouveaux états d'énergie. Ces intermédiaires de réaction qui 
dépendent de la nature du mécanisme réactionnel sont localisés à l'interface mais ils sont plutôt liés soit :

- à la solution. C'est le cas pour les réactions cathodiques qui ne peuvent exister qu'en présence d'espèces rédox au sein de la solution. Les intermédiaires sont alors formés par interaction entre la surface du SC et les espèces arrivant à la surface lors de la première étape de réduction ;

- au solide. C'est le cas pour les réactions anodiques faisant intervenir les atomes du réseau cristallin. Les intermédiaires sont alors créés par un affaiblissement et même la rupture de certaines liaisons en surface par l'action des photoporteurs.

Le taux d'injection obtenu lors de la réaction globale est lié à ces intermédiaires réactionnels et aux niveaux énergétiques qui leur correspondent. Une injection efficace nécessite une position adaptée de ces niveaux :

- proche de la bande de valence lors de la réduction,

- proche de la bande de conduction lors de l'oxydation du matériau,

- en résonance avec l'une ou l'autre des bandes.

L'injection est très faible, voire inexistante lorsque les états sont placés loin, à l'intérieur de la bande interdite, par rapport à la bande concernée par le mécanisme.

Les niveaux susceptibles d'injecter des porteurs majoritaires dépendent de la chimie de surface du matériau.

Réactivité et efficacité de l'injection. - La surface est la zone de rupture du réseau périodique et constitue une zone privilégiée de défauts. A la surface il existe donc des spécificités, que l'on peut associer à des niveaux particuliers impliquant une réactivité différente de celle des atomes au sein du volume. En physique des surfaces des SC, les défauts dépendent étroitement des matériaux. Les lacunes ou les anti-sites [26] jouent un rôle prépondérant. Par analogie, pour les interfaces SC/liquide, on peut penser que le niveau et les caractéristiques des défauts, à l'origine du mécanisme d'injection, dépendent du matériau et de leur environnement chimique qui varie selon le solvant, la concentration des espèces en solution, le $\mathrm{pH}$ du milieu..

On peut alors discuter séparément, à l'appui des remarques précédentes, les résultats obtenus d'une part sur les matériaux de type $\mathrm{p}$ et d'autre part sur les matériaux de type $\mathrm{n}$.

Electrodes de type p. Photoréduction. - Ces réactions correspondent à la réduction de systèmes rédox faisant intervenir au moins deux étapes mono-électroniques. L'injection du porteur majoritaire nécessite deux conditions :

- l'espèce intermédiaire donnant lieu à l'injection de trous doit avoir ses niveaux situés favorablement par rapport à la bande de valence. Ce seul critère thermodynamique ne permet pas d'expliquer les différences de comportement de GaP, GaAs et InP en présence d'oxygène. En effet, on observe un effet doubleur sur p-GaP en présence d'oxygène [14], alors qu'aucun effet n'est obtenu avec p-InP, ce qui n'est pas cohérent avec la position commune des bandes de valence des composés III-P [22].

Ce type de contradiction entre p-GaAs, p-GaP et p-InP est également observé lors de la réduction de l'eau oxygénée [27]. Un effet doubleur est obtenu uniquement avec les électrodes de $p-G a P$ et $p-G a A s$ et seulement pour certaines concentrations en $\mathrm{H}_{2} \mathrm{O}_{2}$. Ceci implique l'existence d'une contribution chimique à l'effet doubleur.

- la probabilité d'injection doit être grande, donc l'interaction entre les espèces en solution et les atomes de surface doit être suffisamment forte. Néanmoins elle doit donner lieu à des processus réversibles, ce qui implique que la surface retrouve sa situation originelle dès que ces espèces sont éliminées. On peut ainsi expliquer l'absence d'effet doubleur sur p-InP en considérant une interaction différente entre le radical $\mathrm{HO}_{2}^{*}$ et les atomes de surface. Cette 
différence d'interaction par rapport à GaAs et GaP peut s'expliquer par les différences de réactivité de InP vis-à-vis des protons plus forte que celle de GaP [28]. Ces derniers étant nécessaires à la formation du radical, on peut raisonnablement expliquer la différence de réactivité par cette hypothèse.

Cette interaction solution-surface semble donc pouvoir être modulée selon la structure de l'espèce qui interagit.

Cet exemple prouve que l'espèce agissante doit avoir une efficacité spécifique puisque, pour un niveau énergétique comparable, elle peut conduire à des niveaux d'injection très différents. Ainsi, contrairement à ce qui est observé pour d'autres rédox comme l'oxygène ou l'eau oxygénée, la réduction du brome conduit à un doublement du photocourant quel que soit le matériau (InP, GaP ou GaAs) [29].

La nature de l'interaction avec le réseau doit donc être considérée comme un paramètre essentiel gouvernant en partie l'efficacité de l'injection.

Electrodes de type n. Photo-oxydation. - Dans ce cas, il existe une interaction forte et irréversible conduisant à la déstructuration du réseau. Mais, comme dans le cas précédent la nature des liaisons entre les différents éléments $\mathrm{Ga}$ ou In d'une part et $\mathrm{P}$ ou As d'autre part dans les intermédiaires de corrosion doit moduler la position énergétique de ces intermédiaires et donc leur probabilité d'injection. La croissance d'un film d'oxyde en surface suivie par sa dissolution est un processus cinétique complexe qui peut conduire à des effets fort différents selon les matériaux.

Le courant d'injection d'électrons ne peut être expliqué que par la présence d'un ou plusieurs états électroniques à la surface du SC. Le processus d'injection d'électrons doit être décrit comme une émission spontanée d'un électron présent sur cet état vers la BC. Ce saut ne peut être thermiquement activé que s'il existe soit un état proche de la BC soit un état résonnant avec la $\mathrm{BC}$. Les phénomènes d'injection ne peuvent être spontanés. Ils nécessitent toujours la présence d'une étape d'excitation pourvoyeuse d'espèces minoritaires $\left(h^{+}\right)$qui déclenche le phénomène de l'oxydation. La situation de non équilibre à l'interface, lorsqu'il existe un enrichissement en trous, conduit à une déstabilisation de l'édifice chimique superficiel.

$$
(\text { III.V })+\mathbf{h}^{+} \rightarrow(\text { III. V })^{+}
$$

L'injection des électrons va prendre naissance dans ces zones déstabilisées, voire transformées, après une interaction avec un agent nucléophile en solution. Plusieurs processus peuvent alors avoir lieu selon la réactivité spécifique des atomes de surface fragilisés et des espèces en solution. La notion de liaison pendante est alors à considérer dans le contexte général de son interaction avec la solution.

La diversité des états intermédiaires est grande et cela peut engendrer des déplacements énergétiques significatifs d'un état à un autre. A chaque stade du processus global il doit y avoir une distribution énergétique caractéristique de l'état de liaison avec le réseau d'une part et avec le solvant d'autre part.

Il existe une corrélation entre l'effet d'injection et la nature de l'anion présent dans le composé. Les composés phosphorés semblent présenter un caractère fortement injectant. Les intermédiaires responsables sont alors fortement liés à la chimie du phosphore. Les espèces phosphorées auront des niveaux énergétiques proches de la $\mathrm{BC}$ ou résonnants avec la surface comme le montrent les résultats obtenus avec InP et GaP. A contrario, ceci implique que pour les espèces intermédiaires associées aux autres éléments $\mathrm{V}$ (As et $\mathrm{Sb}$ ), les positions énergétiques associées doivent se situer très loin dans la bande interdite ce qui semble en accord avec la littérature, à la fois pour le matériau au contact du vide [30, 31] mais aussi in situ pour une jonction SC/électrolyte [32]. Il faut néanmoins considérer ces faits comme une 


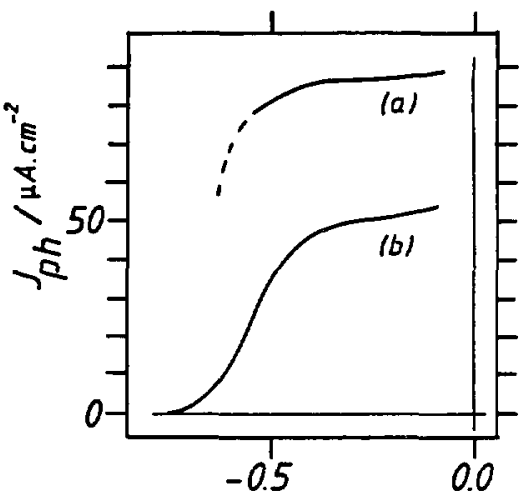

a)

Potential/VvsMSE

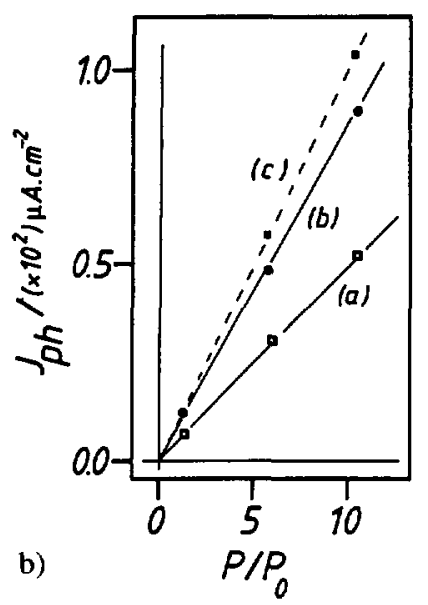

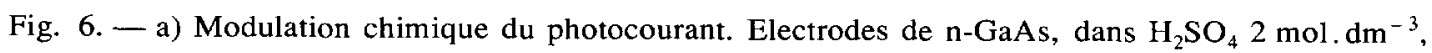
$P=10,4 P_{0}$. a) En présence d'eau oxygénée $\left(C=1 \mathrm{~mol} . \mathrm{dm}^{-3}\right)$. b) Milieu support. b) Photocourant en fonction de la puissance lumineuse $P / P_{0}$. Electrolyte $\mathrm{H}_{2} \mathrm{SO}_{4} 2 \mathrm{~mol} . \mathrm{dm}^{-3}$ a) $\mathrm{GaAs}$ de type n. b) $\mathrm{GaAs}$ de type $\mathrm{n}$ en présence d'eau oxygénée $\left(C=1 \mathrm{~mol} . \mathrm{dm}^{-3}\right)$. c) InP de type $\mathrm{n}$ en présence ou non d'eau oxygénée.

(a) Chemical modulation of photocurrent for n-type GaAs electrodes, $\mathrm{H}_{2} \mathrm{SO}_{4} 2$ mol. $\mathrm{dm}^{-3} P=$ $10.4 P_{0}$. a) In presence of hydrogen peroxide $\left(C=1 \mathrm{~mol} . \mathrm{dm}^{-3}\right)$. b) In acidic medium. b) Photocurrent as a function of the light intensity $P / P_{0}$. a) n-GaAs in supporting electrolyte $\left(\mathrm{H}_{2} \mathrm{SO}_{4} 2 \mathrm{~mol}\right.$. dm $\left.{ }^{-3}\right)$. b) nGaAs in presence of hydrogen peroxide $\left(C=1 \mathrm{~mol} . \mathrm{dm}^{-3}\right)$ c) n-InP with or without hydrogen peroxide.]

simple analogie puisque les positions énergétiques dépendent fortement de l'interaction avec l'environnement chimique.

La transposition des résultats obtenus dans l'ultravide n'a pas de caractère absolu mais permet de donner un élément d'explication de certains processus faisant intervenir des interactions à l'interface solide-liquide.

Modulation chimique de l'injection. - L'idée essentielle permettant d'expliciter les mécanismes d'injection qu'ils soient cathodiques ou anodiques est la même. C'est la structure chimique de l'espèce intermédiaire qui est au centre du mécanisme d'injection. Les niveaux d'énergie asssociés à ces intermédiaires réactionnels peuvent donc être chimiquement modulés selon la nature de l'interaction avec la surface. Toute réaction chimique peut déplacer le niveau énergétique de l'intermédiaire réactionnel et donc moduler le niveau d'injection. Les limites théoriques concernant deux exemples sont données dans les tableaux de la figure 2 .

Deux exemples accréditant cette hypothèse sont donnés dans la figure 6 pour GaAs et dans la figure 7 pour InP.

Les figures $6 a$ et $6 \mathrm{~b}$ montrent qu'il est possible de moduler l'effet d'injection lors de la photocorrosion de n-GaAs. Si l'on ajoute une certaine concentration d'eau oxygénée, le photocourant obtenu sur une électrode de n-GaAs peut être doublé [33]. Ce processus montre une forte probabilité d'injection dans ces circonstances. Il est explicable si l'on tient compte du fait que l'eau oxygénée oxyde fortement le matériau et conduit à la formation d'un film de surface très perturbé cristallographiquement et pouvant piéger certaines espèces intermédiaires très actives ayant un niveau d'énergie en surface proche de la BC. Dans ces conditions, ces 


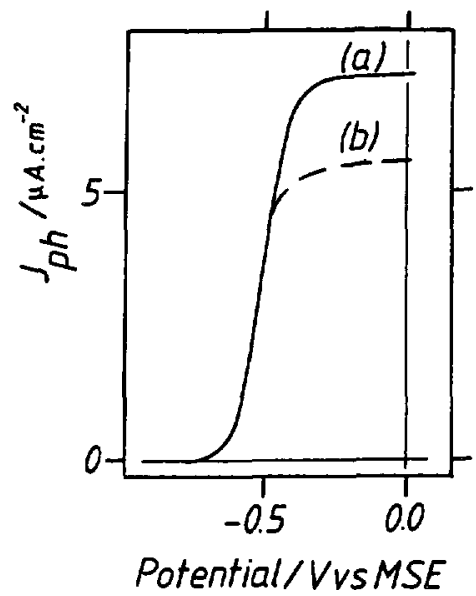

Fig. 7. - Modulation chimique du photocourant. Electrodes de InP de type $\mathrm{n}, P=0,7 P_{0}$. a) Milieu support $\mathrm{H}_{2} \mathrm{SO}_{4} 2 \mathrm{~mol} . \mathrm{dm}^{-3}$ b) En présence de $\mathrm{HF}(5 \%)$.

[Chemical modulation of the photocurrent. n-InP electrodes. $P=0.7 P_{n}$. a) $\mathrm{In}_{2} \mathrm{SO}_{4} 2$ mol. dm ${ }^{-3}$ b) In presence of HF ( $5 \%)$.]

espèces ont alors une grande probabilité d'injection et il est possible d'obtenir une réaction globale d'oxydation de GaAs correspondant à la réaction 4-2-c du tableau II.

La figure 7 est associée au traitement d'une électrode n-InP par une solution d'acide fluorhydrique. On constate que si l'on plonge le matériau dans une solution diluée de HF à $5 \%$, le photocourant diminue considérablement. Il est connu que l'acide HF dissout très rapidement les oxydes d'indium et de phosphore [34]. L'interface électrode/solution en présence de HF doit donc être quasi exempte d'oxyde. Dans ces conditions, aucun film de surface ne se forme lors de l'illumination de l'électrode (ou s'il se forme il se dissout quasi instantanément) et il est alors très difficile aux espèces intermédiaires d'injecter des électrons. Dans ce cas l'effet d'injection est minimisé, ceci peut être dû soit à l'impossibilité d'atteindre un niveau d'énergie suffisant pour pouvoir injecter l'électron, soit à une durée de vie trop brève de l'espèce qui ne permet pas le transfert injectant.

Ces deux exemples montrent la difficulté de conclure au niveau de l'efficacité d'injection. Il semble que le niveau des intermédiaires ait un grand rôle à jouer et que ce niveau énergétique soit directement lié à la réactivité chimique de l'entité (espèce-atome de surface) qui peut être modifiée par des conditions expérimentales telles que le $\mathrm{pH}$, la concentration des espèces, le flux lumineux, la face cristallographique,..

\section{Conclusion.}

Dans cet article, nous avons mis en évidence un certain nombre de résultats importants.

- L'étude précise des phénomènes d'injection est souvent à même de discriminer entre plusieurs types de mécanismes réactionnels.

- L'existence de ces effets démontre la présence d'états électroniques en surface capables d'injecter des porteurs majoritaires. Ce type d'objet est aisément détectable dans les jonctions SC/électrolyte contrairement aux autres types d'interfaces.

- Il est possible de moduler les effets d'injection en modifiant simplement l'environnement chimique. Ceci démontre que les états injectants n'ont pas une structure prédéterminée par la 
nature du matériau, mais doivent plutôt être considérés comme le résultat d'une construction complexe entre les défauts de surface du matériau et les espèces constituant l'environnement chimique qui participe au mécanisme réactionnel. Tout cela nous permet d'affirmer l'importance considérable de la notion de zone superficielle réactionnelle adaptée au mécanisme d'injection. Elle doit répondre à des critères de liaison avec le réseau, de stabilité chimique à l'échelle du temps de l'injection, de position énergétique favorable pour l'émission de porteurs majoritaires au détriment de la capture de porteurs minoritaires au cours de l'étape réactionnelle incriminée.

Ces conclusions sont données dans l'hypothèse d'une surface uniformément concernée par le mécanisme réactionnel. Cependant l'importance de la notion de film superficiel nous permet d'envisager une autre hypothèse considérant une surface non uniforme vis-à-vis du phénomène d'injection qui sera décrite ultérieurement.

\section{Bibliographie}

[1] Morrison S. R., Electrochemistry at semiconductor and oxidized metal electrodes (Plenum press N.Y., 1980).

[2] Pleskov Y. V. and Gurevich Y. Y., Semiconductor Photoelectrochemistry (Consultants Bureau, N.Y., 1986).

[3] Memming R., J. Electrochem. Soc. 116 (1969) 785.

[4] Goosens H. H., Streller K. and Gomes W. P., J. Electroanal. Chem. 286 (1990) 133.

[5] Van den Meerakker J. E. A. M., Electrochim. Acta 35 (1990) 1267.

[6] Peter L. M.. Bonazio A. M., Lewerenz H. V. and Stumper J., J. Electroanal. Chem. 290 (1990) 229.

[7] Kohl P. A., Wolowodiuk C. and Ostermayer Jr F. W., J. Electrochem. Soc. 130 (1983) 2288.

[8] Ellis A. B., Bolts J. M. and Wrighton M. S., J. Electrochem. Soc. 124 (1977) 1603.

[9] Rosamilia J. M., Schneemayer L. F. and Miller B., J. Electrochem. Soc. 134 (1987) 1684.

[10] Vanmaekelbergh D., Kelly J. J., Lingier S. and Gomes W. P., Ber. Bunsenges. Phys. Chem. 92 (1988) 1068.

[11] Vanmaekelbergh D. and Kelly J. J., J. Electrochem. Soc. 136 (1989) 108.

[12] Gerischer H. and Mindt W., Surf. Sci. 4 (1966) 440.

[13] a) Peat R. and Peter L. M., Electrochimica Acta 31 (1986) 731 ;

b) Li J., Peat R. and Peter L. M., J. Electroanal. Chem. 200 (1986) 333.

[14] Li J., Peat R. and Peter L. M., J. Electroanal. Chem. 185 (1985) 339.

[15] Fotouhi B., Triboulet R. and Etman M., J. Electroanal. Chem. 195 (1985) 425.

[16] a) Ellis A. B., Bolts J. M. and Wrighton M. S., J. Electrochem. Soc. 124 (1977) 1603 ;

b) Allongue P. and Cachet H., Electrochimica Acta 33 (1988) 79.

[17] Gartner W. W., Phys. Rev. 116 (1959) 84.

[18] a) Reichman J., Appl. Phys. Lett. 36 (1980) 574 ;

b) Reichman J. and Russak M. A., Photoeffects at semiconductor-electrolytes Interfaces, A. J. Nozik Ed., ACS symposium Seriès $N^{\circ} 146$, Washington (1981).

[19] Reiss H., J. Electrochem. Soc. 125 (1978) 937.

[20] a) Wilson R. H., J. Appl. Phys. 48 (1977) 4292 ;

b) Lemasson P., Etcheberry A. and Gautron J., Electrochim. Acta 27 (1981) 607.

[21] Preusser S., Herlem M., Etcheberry A. and Jaume J., Electrochim. Acta 37 (1992) 289.

[22] Etcheberry A., Gautron J. and Sculfort J. L., J. Electroanal. Chem. 247 (1988) 265.

[23] Allongue P. and Blonkowski S., J. Electroanal. Chem. 316 (1991) 57.

[24] a) Lavagna M., Pique J. P. and Marfaing Y., Solid State Elec. 20 (1977) 235 ;

b) Reichman J., Appl. Phys. Lett. 38 (1981) 251.

[25] Kelly J. J., Miles B. P., Verhaeghe N. A. M., Stumper J. and Peter L. M., Electrochim. Acta 37 (1992) 909. 
[26] Lannoo M. and Friedel J., Atomic and Electronic Structure of Surfaces. Springer Verlag Series in Surface Science 16 Ed. (Cardona, Berlin. 1991).

[27] Memming R., J. Electrochem. Soc. 116 (1969) 785.

[28] a) Madou M. J., Cardon F. and Gomes W. P., Ber. Bunsenges Phys. Chem. 82 (1978) 819 ;

b) Menezes S., Miller B. and Backman K. J., J. Vac. Sci. Technol. B 1 (1983) 48.

[29] Notten P. H. L., Van den Meerakker J. E. A. M. and Kelly J. J., Etching of III-V semiconductors. An electrochemical approach (Elsevier Oxford, 1991).

[30] Allen R. E., Sankey O. F. and Dow J. D., Surf. Sci. 168 (1986) 376.

[31] Spicer W. E., Newman N., Kundelewick T., Petro W. G., Williams M. D., McCants C. E. and Lindau I., J. Vac. Sci. Technol. B 3 (1985) 1178 et toutes les références de cet article concernant GaAs, GaP et InP.

[32] Allongue P. et Cachet H., Electrochim. Acta 33 (1988) 179.

[33] Achard M., Thèse Doctorat Ecole Centrale de Lyon (1991).

[34] Guivarc'h A., L'Haridon M., Pelous G., Hollinger G. and Pertosa P., J. Appl. Phys. 55 (1984) 1139. 